\title{
Weak Values, Quantum Trajectories, and the Stony-Brook Cavity QED experiment
}

\author{
H.M. Wiseman \\ Centre for Quantum Dynamics, School of Science, Griffith University, Brisbane, Queensland 4111, Australia
}

(February 9, 2008)

\begin{abstract}
Weak values as introduced by Aharonov, Albert and Vaidman (AAV) are ensemble average values for the results of weak measurements. They are interesting when the ensemble is preselected on a particular initial state and postselected on a particular final measurement result. I show that weak values arise naturally in quantum optics, as weak measurements occur whenever an open system is monitored (as by a photodetector). I use quantum trajectory theory to derive a generalization of AAV's formula to include (a) mixed initial conditions, (b) nonunitary evolution, (c) a generalized (non-projective) final measurement, and (d) a non-back-action-evading weak measurement. I apply this theory to the recent Stony-Brook cavity QED experiment demonstrating wave-particle duality [G.T. Foster, L.A. Orozco, H.M. Castro-Beltran, and H.J. Carmichael, Phys. Rev. Lett. 85, 3149 (2000)]. I show that the "fractional" correlation function measured in that experiment can be recast as a weak value in a form as simple as that introduced by AAV.
\end{abstract}

03.65.Ta, 42.50.Lc, 42.50.Ct, 42.50.Ar

\section{INTRODUCTION}

The concept of weak values in quantum mechanics was formulated by Aharonov, Albert and Vaidman (AAV) [1] , using the earlier two-wave-function formalism of Ref. [2]. A weak value is the ensemble average value of a weak measurement result. A weak measurement is one that minimally disrupts the system, while consequently yielding a minimal amount of information about the observable measured. For a given initial system state, the ensemble average of weak measurement results is the same as for strong (i.e. projective) measurement results. Where weak measurements are interesting is when a final as well as an initial state is specified. Here the final state is the result of a second measurement (a strong one), so that the ensemble average is taken over a postselected ensemble, in which the desired result for the final measurement was obtained.

Let the desired initial system state be $|\psi\rangle$, and let $\psi$ denote the event that this is successfully prepared. Let the desired final system state be $|\phi\rangle$, and let $\phi$ denote the event that the appropriate result is obtained by the final projective measurement. Let the observable to be measured at an intermediate time have operator $\hat{X}$, and denote the result of the measurement $X_{n}$. Here the nature $n$ of the measurement could be strong $(n=s)$ or weak $(n=w)$. In the former case, which corresponds to a projective measurement, $X_{s}$ reproduces the statistics of $\hat{X}$. In the latter case, $X_{w}$ does not, and indeed is not confined to the spectrum of $\hat{X}$. We do require however that in all cases $X_{n}$ be an unbiased estimator, in the sense that

$$
\left\langle X_{n}\right\rangle_{\psi} \equiv \mathrm{E}\left[X_{n} \mid \psi\right]=\langle\psi|\hat{X}| \psi\rangle
$$

Here E denotes expectation value, and the vertical line denotes "given". The postselected value we desire is

$$
\begin{aligned}
{ }_{\phi}\left\langle X_{n}\right\rangle_{\psi} & \equiv \mathrm{E}\left[X_{n} \mid \psi, \phi\right]=\sum_{x} x \operatorname{Pr}\left[X_{n}=x \mid \phi, \psi\right] \\
& =\frac{\sum_{x} x \operatorname{Pr}\left[\phi \mid X_{n}=x, \psi\right] \operatorname{Pr}\left[X_{n}=x \mid \psi\right]}{\sum_{y} \operatorname{Pr}\left[\phi, X_{n}=y \mid \psi\right]}
\end{aligned}
$$

Here $\operatorname{Pr}[H]$ denotes the probability for event $H$.

For simplicity, assume for the moment that there is no evolution between measurements. Then for a strong intermediate measurement, the postselected average value of $X_{s}$ is

$$
\begin{aligned}
{ }_{\phi}\left\langle X_{s}\right\rangle_{\psi} & =\frac{\sum_{x} x \operatorname{Pr}\left[\phi \mid X_{s}=x\right] \operatorname{Pr}\left[X_{s}=x \mid \psi\right]}{\sum_{y} \operatorname{Pr}\left[\phi \mid X_{s}=y\right] \operatorname{Pr}\left[X_{s}=y \mid \psi\right]} \\
& =\frac{\sum_{x}|\langle\phi \mid x\rangle|^{2} x|\langle x \mid \psi\rangle|^{2}}{\sum_{y}|\langle\phi \mid y\rangle|^{2}|\langle y \mid \psi\rangle|^{2}} .
\end{aligned}
$$

Here $|x\rangle$ denotes an eigenstate of $\hat{X}$. In the final result there is no direct connection between the initial state and the final state. The intermediate strong measurement has destroyed that connection, as it enables the dropping of the conditional $\mid \psi$ from $\operatorname{Pr}\left[\phi \mid X_{s}=x, \psi\right]$ in Eq. (1.3). Basically there are no surprises here.

Contrast this result with that from a weak measurement, as derived by AAV:

$$
{ }_{\phi}\left\langle X_{w}\right\rangle_{\psi}=\operatorname{Re} \frac{\langle\phi|\hat{X}| \psi\rangle}{\langle\phi \mid \psi\rangle} .
$$

Aharonov and Vaidman derive this in detail in Ref. [3], and it is also derived here, in Sec. II, from a somewhat different perspective. This formula appears to have a much more of a mysterious "quantum" nature than Eq. (1.5), as it involves the initial and final states linearly (rather than bilinearly) on the numerator and denominator. As a consequence, weak values can have bizarre properties, especially when the outcome $\phi$ used for postselection is improbable [3]. For example, the weak value ${ }_{\phi}\left\langle X_{w}\right\rangle_{\psi}$ may 
lie outside the range of eigenvalues for $\hat{X}$ [1, [3] . Counterfactual paradoxes [1] can also be expressed and resolved in this language [5.6].

It might be thought that weak values would be mainly of theoretical interest, because arbitrarily weak measurements would not arise naturally in experiments [7]. But in fact arbitrarily weak measurements arise all the time, whenever a system is monitored (i.e. measured continuously in time). The measurement theory for monitored systems was developed by mathematical physicists [8 [11]. It was subsequently rediscovered by workers in quantum optics 12 14. when it was found necessary to describe the conditioning of systems on an observed photocurrent. In quantum optics, the theory of continuous monitoring, especially for homodyne and heterodyne measurement, has come to be known as quantum trajectory theory 14.15 .

In this paper I show in Sec. II how the AAV formula can be derived using the approach of quantum trajectory theory. I derive a simple generalization arising from removing the restriction that the weak measurement of $X$ be a quantum non-demolition measurement. In Sec. III I derive a more complete generalization which allows for (a) mixed initial conditions; (b) non-unitary evolution between measurements; and (c) a non-projective final measurement. Within this context, the AAV formula can be seen to be a special case of the correlation functions that have been used in quantum optics for a long time [16]. Finally, in Sec. IV I show that the correlation function measured in the recent cavity-QED experiment at Stony-Brook [17] can be reformulated as a weak value almost exactly as originally proposed by AAV.

\section{WEAK VALUES: THE SPECIAL THEORY}

Monitoring a quantum system can be treated by considering a sequence of discrete weak measurements, each taking time $\delta t$, and letting $\delta t \rightarrow d t$. To obtain a sensible limit, the strength of each measurement must scale as $\delta t$. By this I mean that the post-measurement system state should, on average, be different from the premeasurement state by an amount of order $\delta t$.

To describe weak measurements, we require generalized (non-projective) quantum measurement theory [18 20]. Let the measurement result be the random variable $X_{n}$ as above. In the case of efficient measurements, the measurement can be described in terms of a set of measurement operators $\left\{\hat{M}_{x}\right\}_{x}$, each associated with a result $X_{n}=x$. The post-measurement conditioned state is given by

$$
\rho_{x}(t+\delta t)=\frac{\hat{M}_{x} \rho(t) \hat{M}_{x}^{\dagger}}{\operatorname{Pr}\left[X_{n}=x\right]},
$$

where the probability for the result is

$$
\operatorname{Pr}\left[X_{n}=x\right]=\operatorname{Tr}\left[\hat{M}_{x} \rho(t) \hat{M}_{x}^{\dagger}\right] .
$$

By conservation of probability, the measurement operators must obey a completeness relation

$$
\sum_{x} \hat{E}_{x}=\hat{1},
$$

where $\hat{E}_{x}=\hat{M}_{x}^{\dagger} \hat{M}_{x}$ is known as the probability operator or effect for the result $x$.

As above, we require the measurement of $\hat{X}$ to be unbiased, so that $\left\langle X_{n}(t)\right\rangle_{\rho(t)}=\operatorname{Tr}[\rho(t) \hat{X}]$. This is satisfied by the following measurement operator, which also has a suitably weak effect on the system [21]

$$
\hat{M}_{x}=(2 \pi / \delta t)^{-1 / 4} \exp \left(-x^{2} \delta t / 2\right)\left[1+\delta t\left(x \hat{c}-\hat{c}^{\dagger} \hat{c} / 2\right)\right] .
$$

Here $\hat{c}$ is an operator such that

$$
\hat{c}+\hat{c}^{\dagger}=\hat{X} .
$$

For convenience we are measuring time in units such that $\hat{c}^{\dagger} \hat{c} \delta t$ is dimensionless. Note that by allowing $\hat{c}$ to be nonHermitian we are allowing for non-back-action-evading measurements of $\hat{X}$, even ignoring any evolution between measurements.

The measurement operators satisfy a continuous version of the completeness relation $(2.3)$ to order $(\delta t)^{2}$ :

$$
\int d x \hat{M}_{x}^{\dagger} \hat{M}_{x}=\hat{1}+O\left(\delta t^{2}\right),
$$

In the limit $\delta t \rightarrow d t$, the correction vanishes and it is not difficult to show 21] that the measurement result $X_{w}$ has statistics given by

$$
X_{w}(t)=\operatorname{Tr}[\hat{X} \rho(t)]+\xi(t) .
$$

Here is a Gaussian white noise term, defined by $\xi(t)=$ $d W(t) / d t$ where

$$
\begin{aligned}
\mathrm{E}[d W(t)] & =0, \\
d W(t)^{2} & =d t .
\end{aligned}
$$

Moreover, the stochastic post-measurement conditioned state is given by

$$
\rho_{X_{w}}(t+d t)=\{1+\mathcal{D}[\hat{c}] d t+\mathcal{H}[\hat{c}] d W(t)\} \rho(t) .
$$

Here for arbitrary operators $\hat{A}$ and $\hat{B}$

$$
\begin{aligned}
\mathcal{D}[\hat{A}] \hat{B} & \equiv \hat{A} \hat{B} \hat{A}^{\dagger}-\frac{1}{2}\left\{\hat{A}^{\dagger} \hat{A}, \hat{B}\right\}, \\
\mathcal{H}[\hat{A}] \hat{B} & \equiv(\hat{A}-\operatorname{Tr}[\hat{A} \hat{B}]) \hat{B}+\text { H.c.. }
\end{aligned}
$$

Averaging over the measurement results would give

$$
d \rho=d t \mathcal{D}[\hat{c}] \rho,
$$

which does not necessarily preserve $\langle\hat{X}\rangle_{\rho(t)}=\operatorname{Tr}[\hat{X} \rho(t)]$ because $\hat{c}$ need not commute with $\hat{X}$. 
For continuous measurements we wish to consider a sequence of measurements as described above. However, for the purposes of deriving weak values, we wish to assume we have just a single such weak measurement, at time $t$. (This unrealistic assumption will be removed in Sec. III.) Say the system was prepared at time 0 in state $|\psi(0)\rangle$ and evolved unitarily to state $|\psi\rangle$ at time $t$. Let us use $\rho(t)$ for $|\psi\rangle\langle\psi|$. The infinitely weak (i.e. infinitesimally strong) measurement is then performed [22]. Then at time $T$ a final projective measurement is made, and we wish to keep only those values of $X$ obtained for which the final result corresponds to the state $|\phi(T)\rangle$. Let $|\phi\rangle$ be that state evolved unitarily backwards in time from $T$ to just after $t$. Dropping the time arguments, we have in the notation of Sec. I,

$$
\begin{aligned}
{ }_{\phi}\left\langle X_{w}\right\rangle_{\psi} & =\mathrm{E}\left[X_{w} \mid \phi, \psi\right]=\sum_{x} x \operatorname{Pr}\left[X_{w}=x \mid \phi, \psi\right] \\
& =\sum_{x} x \operatorname{Pr}\left[X_{w}=x, \phi \mid \psi\right] / \operatorname{Pr}[\phi \mid \psi]
\end{aligned}
$$

Here we have used a sum rather than an integral merely for convenience.

Now because the measurement is infinitely weak, it does not affect the denominator $D=\operatorname{Pr}[\phi \mid \psi]$, which equals $|\langle\phi \mid \psi\rangle|^{2}$. The numerator meanwhile evaluates to

$$
\begin{aligned}
N & =\sum_{x} x \operatorname{Pr}\left[\phi \mid X_{w}=x, \psi\right] \operatorname{Pr}\left[X_{w}=x \mid \psi\right] \\
& =\sum_{x} x\left\langle\phi\left|\rho_{x}(t+d t)\right| \phi\right\rangle \operatorname{Pr}\left[X_{w}=x \mid \psi\right] \\
& =\mathrm{E}\left[X_{w}\left\langle\phi\left|\rho_{X_{w}}(t+d t)\right| \phi\right\rangle\right] .
\end{aligned}
$$

Now from the above formulae (2.7)-(2.10) it is easy to see that this evaluates to

$$
\left\langle\phi\left|\left\{\rho(t)\langle\hat{X}\rangle_{\rho(t)}+\mathcal{H}[\hat{c}] \rho(t)\right\}\right| \phi\right\rangle+O(d t) .
$$

Expanding the superoperator $\mathcal{H}$ and ignoring infinitesimals gives

$$
\left\langle\phi\left|\left\{\hat{c} \rho(t)+\rho(t) \hat{c}^{\dagger}\right\}\right| \phi\right\rangle .
$$

Substituting this into Eq. (2.15), and using $\rho(t)=|\psi\rangle\langle\psi|$, gives

$$
\begin{aligned}
{ }_{\phi}\left\langle X_{w}\right\rangle_{\psi} & =\frac{\langle\phi|\hat{c}| \psi\rangle\langle\psi \mid \phi\rangle+\text { H.c. }}{|\langle\phi \mid \psi\rangle|^{2}} \\
& =2 \operatorname{Re} \frac{\langle\phi|\hat{c}| \psi\rangle}{\langle\phi \mid \psi\rangle} .
\end{aligned}
$$

In the case of QND measurements where $\hat{c}=\hat{X} / 2$, this reduces to the AAV formula (1.6). Putting in the unitary time evolution explicitly, and setting $T=t+\tau$,

$$
{ }_{\phi}\left\langle X_{w}(t)\right\rangle_{\psi}=2 \operatorname{Re} \frac{\langle\phi(T)|\hat{U}(\tau) \hat{c} \hat{U}(t)| \psi(0)\rangle}{\langle\phi(T)|\hat{U}(T)| \psi(0)\rangle} .
$$

\section{WEAK VALUES: THE GENERAL THEORY}

We now generalize the above theory by removing many of the assumptions. First, we allow the initial system state $\rho(0)$ to be mixed. Second, we allow arbitrary Markovian evolution [20] of $\rho$ between measurements:

$$
\dot{\rho}=\mathcal{L} \rho \equiv-i[\hat{H}, \rho]+\sum_{\mu} \mathcal{D}\left[\hat{c}_{\mu}\right]
$$

Here $\hat{H}$ is an Hermitian operator while $\left\{\hat{c}_{\mu}\right\}_{\mu}$ is a set of arbitrary operators (which strictly should be bounded [24). It would be natural for one of them to be the operator $\hat{c}$ introduced in Sec. II. Then at any time between preparation and final measurement, the conditional evolution of the system would be

$$
d \rho_{X_{w}(s)}=d s \mathcal{L} \rho+d W(s) \mathcal{H}[\hat{c}] \rho .
$$

Finally, we allow for the final measurement at time $T$ to be described by a positive operator $\hat{E}(T)$ which is not necessarily a projector $|\phi(T)\rangle\langle\phi(T)|$. As long as $\hat{1}-\hat{E}(T) \geq 0, \hat{E}(T)$ is an effect for some final measurement.

Using $E$ and $\rho$ to denote the events of successful preparation and successful final measurement, the weak value at time $t$ we wish to calculate now is

$$
\begin{aligned}
{ }_{E}\left\langle X_{w}\right\rangle_{\rho} & =\mathrm{E}\left[X_{w} \mid E, \rho\right]=\sum_{x} x \operatorname{Pr}\left[X_{w}=x \mid E, \rho\right] \\
& =\sum_{x} x \operatorname{Pr}\left[X_{w}=x, E \mid \rho\right] / \operatorname{Pr}[E \mid \rho] .
\end{aligned}
$$

As before, the denominator is unaffected by the weak measurement and is given by

$$
D=\operatorname{Tr}\left[\hat{E}(T) e^{\mathcal{L} T} \rho(0)\right]
$$

The numerator can be manipulated similarly to above to yield

$$
\begin{aligned}
N & =\mathrm{E}\left[X_{w}(t) \operatorname{Tr}\left[\hat{E}(T) e^{\mathcal{L} \tau} \rho_{X_{w}}(t+d t)\right]\right. \\
& =\operatorname{Tr}\left[\hat{E}(T) e^{\mathcal{L} \tau}\left\{\hat{c} e^{\mathcal{L} t} \rho(0)+\text { H.c. }\right\}\right] .
\end{aligned}
$$

Equation (2.23) thus generalizes to

$$
{ }_{E}\left\langle X_{w}(t)\right\rangle_{\rho}=2 \operatorname{Re} \frac{\operatorname{Tr}\left[\hat{E}(T) e^{\mathcal{L} \tau}\left\{\hat{c} e^{\mathcal{L} t} \rho(0)\right\}\right]}{\operatorname{Tr}\left[\hat{E}(T) e^{\mathcal{L} T} \rho(0)\right]} .
$$

The above result looks more like a normalized correlation function, as is familiar in quantum optics, than the unexpected AAV formula (1.6). Indeed, it can be written in the Heisenberg picture 23] as

$$
{ }_{E}\left\langle X_{w}\right\rangle_{\rho}=\frac{\left\langle\hat{E}(T) \hat{c}(t)+\hat{c}^{\dagger}(t) \hat{E}(T)\right\rangle}{\langle\hat{E}(T)\rangle},
$$


with initial conditions supplied by $\rho(0)$ and the bath state implied by the master equation (3.1). However, we can formulate Eq. (3.8) more like Eq. (1.6) as follows. First we denote, in analogy to $|\psi\rangle=U(t)|\psi(0)\rangle$,

$$
\rho=e^{\mathcal{L} t} \rho(0) \text {. }
$$

This is the usual forward time evolution by the master equation (3.1). Next we define a retrodictive [25] positive operator $E$ according to

$$
\operatorname{Tr}[\hat{E} \hat{A}] \equiv \operatorname{Tr}\left[\hat{E}(T) e^{\mathcal{L} \tau} \hat{A}\right],
$$

where $\hat{A}$ is an arbitrary operator.

It is easy to verify that this $\hat{E}$ is $\hat{E}(T)$ evolved forward by time $\tau$ according to the retrodictive master equation

$$
\frac{d \hat{E}}{d t}=+i[\hat{H}, \hat{E}]+\sum_{\mu}\left[\hat{c}_{\mu}^{\dagger} \hat{E} \hat{c}_{\mu}-\frac{1}{2}\left\{\hat{c}_{\mu}^{\dagger} \hat{c}_{\mu}, \hat{E}\right\}\right]
$$

Note that this differs slightly from the retrodictive master equation in Ref. 26. This is because we do not require the norm of $\hat{E}$ to be preserved, as it appears in both the numerator and denominator of Eq. (3.8). That Eq. 3.12) does generate a positive operator is obvious from the solution as a "Dyson-like" sum constructed in the manner of Eq. (7.24) of Ref. 114. Using these definitions, we can write the postselected weak value as

$$
{ }_{E}\left\langle X_{w}\right\rangle_{\rho}=2 \operatorname{Re} \frac{\operatorname{Tr}[\hat{E} \hat{c} \rho]}{\operatorname{Tr}[\hat{E} \rho]} .
$$

Thus retrodiction, quantum trajectories and weak values are all united in this expression.

\section{WEAK VALUES IN THE STONY-BROOK CAVITY QED EXPERIMENT}

The preceding section showed that postselected weak values can be thought of as a correlation function (3.9). In quantum optics weak measurements of the type described in Sec. II are realized through homodyne detection [14,15], where $\hat{c}$ is proportional to the lowering operator of the radiating system. The typical correlation functions thus found are of the form (in the Heisenberg picture)

$$
\begin{aligned}
\langle: \hat{X}(t+\tau) \hat{X}(t):\rangle= & \left\langle\hat{c}(t+\tau) \hat{c}(t)+\hat{c}^{\dagger}(t+\tau) \hat{c}(t)\right. \\
& \left.+\hat{c}^{\dagger}(t) \hat{c}(t+\tau)+\hat{c}^{\dagger}(t) \hat{c}^{\dagger}(t+\tau)\right\rangle .
\end{aligned}
$$

Usually they are evaluated at steady state (ss) where

$$
\rho(0)=\rho_{\mathrm{ss}} \propto \operatorname{nullspace}(\mathcal{L}) .
$$

Note that this is not of the form of Eq. (3.9), so that this correlation function cannot be thought of as a weak value in the AAV sense.
A notable exception to this typicality is the recent cavity QED experiment conducted at Stony-Brook [17]. This involved a damped cavity, very weakly driven, through which a beam of resonant atoms passed. The master equation for the system can be approximated as

$$
\begin{aligned}
\dot{\rho}= & \varepsilon\left[\hat{a}^{\dagger}-\hat{a}, \rho\right]+2 \kappa \mathcal{D}[\hat{a}] \rho \\
& +\sum_{j}^{N}\left\{g_{j}\left[\hat{a}^{\dagger} \hat{\sigma}_{j}^{\dagger}-\hat{\sigma}_{j}^{\dagger} \hat{a}, \rho\right]+2 \gamma_{\perp} \mathcal{D}\left[\hat{\sigma}_{j}\right] \rho\right\} .
\end{aligned}
$$

Here $\hat{\sigma}_{j}=|g\rangle_{j}\langle e|$ is the lowering operator for the $j$ th atom, and $\hat{a}$ is the annihilation operator for the field. The output from the cavity is split into two beams by an 85-15 beam splitter, One beam is detected by homodyne detection (with nett efficiency $\eta_{h}<85 \%$ ), and the other is detected using a photon counter (with nett efficiency $\eta_{c}<15 \%$ ).

Choosing the slightly odd convention of measuring time in units of $1 /\left(2 \kappa \eta_{h}\right)$, the homodyne measurement gives a current proportional to

$$
X_{w}(t)=\operatorname{Tr}[\rho(t) \hat{X}]+\xi(t)
$$

where $\hat{X}=\hat{a}+\hat{a}^{\dagger}$. It conditions the system state $\rho$ by adding the term

$$
d W(t) \mathcal{H}[\hat{a}] \rho
$$

to $d \rho$. Thus the homodyne measurement gives a continuous weak measurement as in Sec. II, with $\hat{c}=\hat{a}$. The photon counting also gives a continuous measurement that is weak in the sense that the average change in the conditioned system in time $\delta t$ is of order $\delta t$. However, unlike homodyne detection, sometimes the change is great. This can be seen from the set of effects $\left\{\hat{E}_{0}, \hat{E}_{1}\right\}$ describing this measurement:

$$
\begin{aligned}
& \hat{E}_{0}=1-\delta t \kappa \eta_{c} \hat{a}^{\dagger} \hat{a}, \\
& \hat{E}_{1}=\delta t \kappa \eta_{c} \hat{a}^{\dagger} \hat{a}
\end{aligned}
$$

These give the probabilities for detecting zero or one photon over a short time $\delta t$. The latter result reveals a lot about the system, and its effect (and measurement operator) are not close to unity.

In the Stony-Brook experiment, the correlation between the homodyne current $X_{w}(t)$ and the photon count increment at time $T=t+\tau$ were measured in order to demonstrate an aspect of wave-particle duality. In the experiment $\tau$ ranged from negative to positive, and the measured correlation was proportional to (in the Heisenberg picture)

$$
h(t-T)=\frac{\left\langle:\left(\hat{a}^{\dagger} \hat{a}\right)(T) \hat{X}(t):\right\rangle_{\mathrm{ss}}}{\left\langle\hat{a}^{\dagger} \hat{a}\right\rangle_{\mathrm{ss}}}
$$


This is a "fractional" (3/2) order correlation function [in contrast to the first order function in Eq. (4.1)]. Here we are interested only in the case $\tau>0$ in which case the correlation function can be written as [27]

$$
h(-\tau)=\frac{\operatorname{Tr}\left[\hat{E}_{1}(T) e^{\mathcal{L} \tau}\left(\hat{a} \rho_{\mathrm{ss}}+\rho_{\mathrm{ss}} \hat{a}^{\dagger}\right)\right]}{\operatorname{Tr}\left[\hat{E}_{1}(T) \rho_{\mathrm{ss}}\right]} .
$$

Defining $\hat{E}_{1}$ to be $\hat{E}_{1}(T)$ retrodicted (as in Sec. III) from time $T$ to time $t$, we can rewrite this correlation function as

$$
{ }_{E_{1}}\left\langle X_{w}\right\rangle_{\rho_{\mathrm{ss}}}=2 \operatorname{Re} \frac{\operatorname{Tr}\left[\hat{E}_{1} \hat{a} \rho_{\mathrm{ss}}\right]}{\operatorname{Tr}\left[\hat{E}_{1} \rho_{\mathrm{ss}}\right]}
$$

It is now clear that the correlation function measured in Ref. [17] is a weak value, preselected by the system being in its stationary state, and postselected on the the final measurement result $E_{1}$ (a photon detected at time $T)$. Actually, since what was measured was a correlation function (i.e. a function of $\tau$ ), this experiment showed the dynamics of a weak value over time. In this case, the spectrum of $\hat{X}$ is the real line, so there is no chance of observing a weak value outside of this range. Nevertheless, the weak values measured in the experiment were, for a range of times $\tau$, very far away from the stationary average value of $\hat{X}$. This lead to the violation of various classical inequalities [28,17]. In hindsight, the strangeness of the weak values in this experiment is not surprising, since the condition cited by Aharonov and Vaidman [3] is fulfilled. That is, the postselection is done on a rare event (the detection of one photon rather than zero photons).

There are a number of features of the above system that let us transform the generalized weak value (4.10) into a form almost identical to the special form (2.22) derived in Sec. II. Because the driving is very weak $\left(\epsilon \ll \kappa, \gamma_{\perp}\right)$, the "jump" terms in the master equation (4.3) have a very small effect. This means that it is possible to approximate the Liouvillian as

$$
\mathcal{L}=\mathcal{H}\left[-i \hat{H}_{\mathrm{eff}}\right]
$$

where

$$
\begin{aligned}
-i \hat{H}_{\mathrm{eff}}= & \varepsilon\left(\hat{a}^{\dagger}-\hat{a}\right)-\kappa \hat{a}^{\dagger} \hat{a} \\
& +\sum_{j}^{N}\left[g\left(\hat{a}^{\dagger} \hat{\sigma}_{j}^{\dagger}-\hat{\sigma}_{j}^{\dagger} \hat{a}\right)-\gamma_{\perp} \hat{\sigma}_{j}^{\dagger} \hat{\sigma}_{j}\right] .
\end{aligned}
$$

Ignoring normalization, this generates nonunitary evolution according to the operator

$$
\hat{N}(t)=\exp \left(-i \hat{H}_{\mathrm{eff}} t\right)
$$

The stationary solution is, to a very good approximation, pure 29.

$$
\rho_{\mathrm{ss}}=\left|\psi_{\mathrm{ss}}\right\rangle\left\langle\psi_{\mathrm{ss}}\right|
$$

where $\left|\psi_{\mathrm{ss}}\right\rangle$ is the eigenstate of $-i \hat{H}_{\mathrm{eff}}$ with eigenvalue having the largest real part.

These results mean that we can approximate Eq. (4.10) by

$$
E_{1}\left\langle X_{w}\right\rangle_{\psi_{\mathrm{ss}}}=2 \operatorname{Re} \frac{\left\langle\psi_{\mathrm{ss}}\left|\hat{a}^{\dagger} \hat{a} \hat{N}(\tau) \hat{a}\right| \psi_{\mathrm{ss}}\right\rangle}{\left\langle\psi_{\mathrm{ss}}\left|\hat{a}^{\dagger} \hat{a} \hat{N}(\tau)\right| \psi_{\mathrm{ss}}\right\rangle}
$$

Again because of the weak excitation, $\left|\psi_{\text {ss }}\right\rangle$ is, to a good approximation, given by

$$
\left|\psi_{\mathrm{ss}}\right\rangle=|0\rangle+\alpha|1\rangle+\beta\left|1^{\prime}\right\rangle .
$$

Here $\alpha$ and $\beta$ scale with $\epsilon$. The state $|0\rangle$ is the state with no excitations, $|1\rangle$ that with one photon but all atoms in the ground state, and $\left|1^{\prime}\right\rangle$ a state with one collective atomic excitation but no photons. The same sort of expansion holds for $\hat{a}\left|\psi_{\mathrm{ss}}\right\rangle / \sqrt{\left\langle\hat{a}^{\dagger} \hat{a}\right\rangle_{\mathrm{ss}}}$ (although the presence of a nonzero $|1\rangle$ term here indicates that $\left|\psi_{\mathrm{ss}}\right\rangle$ does have higher order terms). Furthermore, to leading order, $\hat{N}(t)$ preserves this expansion (apart from normalization). For these reasons, we can replace $\hat{a}^{\dagger} \hat{a}$, which is really $\hat{a}^{\dagger} \hat{a} \otimes \hat{1}_{\text {atoms }}$, by $|1\rangle\langle 1|$. That is, postselection on the detection of a photon is equivalent to postselection on projection into the state $|1\rangle$. The final result thus is

$$
{ }_{E_{1}}\left\langle X_{w}\right\rangle_{\psi_{\mathrm{ss}}}=2 \operatorname{Re} \frac{\left\langle 1|\hat{N}(\tau) \hat{a}| \psi_{\mathrm{ss}}\right\rangle}{\left\langle 1|\hat{N}(\tau)| \psi_{\mathrm{ss}}\right\rangle} .
$$

This is completely analogous Eq. (1.6) when we recognize $N^{\dagger}(\tau)|1\rangle$ as the unnormalized retrodicted final state.

When $\left|\psi_{\text {ss }}\right\rangle$ and $\hat{N}(\tau)$ are substituted into Eq. (4.17), and only the terms of leading order in the driving $\epsilon$ are kept, the result is

$$
\frac{E_{1}\left\langle X_{w}\right\rangle_{\psi_{\mathrm{ss}}}}{\left\langle\psi_{\mathrm{ss}}|\hat{X}| \psi_{\mathrm{ss}}\right\rangle}=1+\zeta e^{-\eta \tau}\left[\cos \Omega \tau+\frac{\eta}{\Omega} \sin \Omega \tau\right]
$$

where $\eta=\left(\kappa+\gamma_{\perp}\right) / 2$ and where $\zeta$ and $\Omega$ are functions of $\kappa, \gamma_{\perp}$, and the coupling coefficients $\left\{g_{j}\right\}_{j}$. This is exactly the same as the analytical result in Ref. [17], where this result was derived from considering the correlation function (4.8) with $T<t$. That is, with the photodetection preceding the homodyne measurement. In that case, the correlation function is

$$
h(-\tau)=h(|\tau|)=\frac{\operatorname{Tr}\left[\hat{X} e^{\mathcal{L}|\tau|} \hat{a} \rho_{\mathrm{ss}} \hat{a}^{\dagger}\right]}{\operatorname{Tr}\left[\hat{a} \rho_{\mathrm{ss}} \hat{a}^{\dagger}\right]} .
$$

In Ref. 17] the symmetry of $h(\tau)$ was established by assuming Gaussian fluctuations. The analysis in this paper shows that the symmetry (to leading order) of $h(\tau)$ can be demonstrated explicitly by calculating both cases. The case of negative $\tau$ is found simply from the usual predictive quantum mechanics with wavefunction collapse 29], and the case of positive $\tau$ equally simply by retrodictive quantum mechanics with weak values, as here.

Finally, it is important to point out that the above form for the weak value as a function of time (4.18) 
matches well with the experiment [17]. This is so even though the experimental conditions are far from ideal, with fluctuations in the number of atoms, transverse motion of the atoms, imperfect beam alignment and so on. This shows that a weak value is not as fragile as one might have thought, and does give quantitative predictions for the behaviour of the system.

\section{DISCUSSION}

In this paper I have combined quantum trajectory theory, and the concepts of weak values and retrodiction in order to show the relation between weak values and certain correlation functions as can be measured in quantum optics. This required a generalization of the weak value theory of Aharonov, Albert and Vaidman. However, in a special case of considerable interest, the Stony-Brook wave-particle cavity QED experiment, I show that the correlation function reduces to a form as simple, and almost the same, as that originally derived by AAV. Moreover, this experiment measured not a weak value at a particular time, but the change in a weak value over time. That is, it showed the dynamics of a weak value.

Weak values are of considerable intrinsic theoretical and philosophical interest. Moreover, the fact that they obey certain well-defined rules [3]5] imply that they are also heuristic tools for predicting the results of experiments. Of course the results could be obtained by other, more laborious methods. But the power of having several fonts of intuition should not be undervalued. The analysis of Sec. IV is a case in point. This work should help to identify other instances in which the theory of weak values can be used in quantum optics and related areas. It may even suggest new experiments that provide further illustrations and applications for this thought-provoking area of quantum physics.

[1] Y. Aharonov, D.Z. Albert, and L. Vaidman, Phys. Rev. Lett 60, 1351 (1988).

[2] Y. Aharonov, O.G. Bergman, and J.L. Lebowitz, Phys. Rev. B 134, 1410 (1964).

[3] Y. Aharonov and L. Vaidman, Phys. Rev. A 41, 11 (1990).

[4] L. Hardy, Phys. Rev. Lett. 68, 2981 (1992).

[5] Y. Aharonov et al., quant-ph/0104062.

[6] K. Mølmer, quant-ph/0109042.
[7] Although, by deliberately constructing a weak measurement, the unusual predictions of AAV [1] were experimentally verified not long after they were proposed, by N.W.M. Ritchie, J.G. Story, and R.G. Hulet, Phys. Rev. Lett. 66, 1107 (1991).

[8] V.P. Belavkin, "Nondemolition measurement and nonlinear filtering of quantum stochastic processes", pp. 245-66 of A. Blaquière (ed.), Lecture Notes in Control and Information Sciences 121 (Springer, Berlin, 1988).

[9] V.P. Belavkin and P. Staszewski, Phys. Rev. A 45, 1347 (1992).

[10] A. Barchielli, Quantum Opt. 2, 423 (1990).

[11] A. Barchielli, Int. J. Theor. Phys. 32, 2221 (1993).

[12] J. Dalibard, Y. Castin and K. Mølmer, Phys. Rev. Lett. 68, 580 (1992).

[13] C.W. Gardiner, A.S. Parkins, and P. Zoller, Phys. Rev. A 46, 4363 (1992).

[14] H.J. Carmichael, An Open Systems Approach to Quantum Optics (Springer-Verlag, Berlin, 1993).

[15] H.M. Wiseman and G.J. Milburn, Phys. Rev. A 47, 1652 (1993).

[16] D.F. Walls and G.J. Milburn, Quantum Optics (Springer, Berlin, 1994), and references therein.

[17] G.T. Foster, L.A. Orozco, H.M. Castro-Beltran, and H.J. Carmichael, Phys. Rev. Lett. 85, 3149 (2000).

[18] K. Kraus, States, Effects, and Operations: Fundamental Notions of Quantum Theory (Springer, Berlin, 1983).

[19] V.B. Braginsky and F.Y. Khalili, Quantum Measurement (Cambridge University Press, Cambridge, 1992).

[20] C.W. Gardiner and P. Zoller, Quantum Noise (Springer, Berlin, 2000).

[21] H.M. Wiseman and L. Diósi, J. Chem. Phys. 268, 91 (2001). quant-ph/0012016

[22] Of course in a real experiment, such as Ref. [17], the measurement is not infinitely weak, otherwise an infinitely large ensemble would be required to obtain a sensible average. The infinitesimal $d t$ is actually a finite but small $\delta t=1 / B$, where $B$ is the bandwidth of the apparatus (including the detector, amplifier, and often a deliberately introduced filter).

[23] Note that $\hat{E}(T)$ in the Heisenberg picture is not necessarily the same as $\hat{E}(T)$ in the Schrödinger picture.

[24] G. Lindblad, Commun. Math. Phys. 48, 199 (1976).

[25] S. M. Barnett, D. T. Pegg, and J. Jeffers, J. Mod. Opt. 47, 1779 (2000).

[26] S.M. Barnett, D.T. Pegg, J. Jeffers, and O. Jedrkiewicz, Phys. Rev. Lett. 86, 2455 (2001).

[27] Note that the $\tau$ I am using is the negative of the $\tau$ in Ref. 17], which is why I write their correlation function as $h(-\tau)$.

[28] H.J. Carmichael, H.M. Castro-Beltran, G.T. Foster, and L.A. Orozco, Phys. Rev. Lett. 85, 1855 (2000).

[29] H.J. Carmichael, R.J. Brecha, and P.R. Rice, Opt. Comm. 82, 73 (1991). 